\title{
Method for measurement of the angles of a tetragonal or corner cube prism
}

\author{
S. Madhusudana Rao \\ Indian Institute of Science \\ Department of Instrumentation \\ Bangalore 560012, India
}

\begin{abstract}
A simple technique is devised to measure the angles of tetragonal prisms without using expensive spectrometers, or autocollimators and angle gauges. (c) 2002 Society of Photo-Optical Instrumentation Engineers. [DOI: 10.1117/1.1481511]

Subject terms: prisms; metrology.
\end{abstract}

Paper 010454 received Dec. 13, 2001; accepted for publication Feb. 1, 2002.

\section{Introduction}

Several methods are currently used to test prism angles. ${ }^{1-6}$ Prisms of all angles can be tested by precision spectrometers and angle dekkors (autocollimators). In normal practice, prism angles are compared by an angle dekkor against a master angle gauge or against a combination of angle gauges. In this method a suitable angle gauge or angle gauge combination is essential to conduct the experiments. Alternate methods are suggested in Refs. 7-13 for circumstances where an autocollimator and angle gauges are not available. In these methods, the angular deviation from symmetry of two surfaces with respect to a side as a base line is determined from the measurement of the screen distance and the separation of the reflected laser spots on a screen before and after the rotation of a partially or fully polished optical component by $180 \mathrm{deg}$. In the case of tetragonal prisms, the 54-deg, 44-min, 8-sec angle needs a suitable angle gauge or a gauge combination, which is very difficult to get from angle gauge manufacturers.

A simple method is suggested for setting up a reference standard for a 54-deg, 44-min, 8-sec angle and also for measuring all the angles of a tetragonal prism.

\section{Principles of the Method}

A tetragonal prism, also known as a corner cube, retroreflector, or as triple mirrors, is shown in Fig. 1. The prism may be regarded as one corner of a glass cube. All the angles at the corner point $O$ are right angles, and the sides $S_{1}, S_{2}$, and $S_{3}$ are all equal. The fourth surface (entrance and exit face for the light beam) makes an angle of 54-deg, 44-min, 8-sec with the other three surfaces of the tetragonal prism.

\subsection{Right Angle}

Figure 2 illustrates the principles of measurement of the 90-deg angle of a prism. The detailed description of these experiments can be found in Refs. 8, 11, and 12 .

If $\alpha$ is the error in the $90-\mathrm{deg}$ angle, It is given by

$\alpha=\frac{A B}{4 O C}$.

\subsection{4-deg, 44-min, 8-sec Angle}

For measuring the 54-deg, 44-min, 8-sec angle, we have made an arrangement for a reference surface using three right-angle prisms and a plane parallel plate, as shown in Fig. 3. For making such an arrangement we have fabricated three right-angle prisms, $P_{1}, P_{2}$, and $P_{3}$ of suitable thicknesses. Prism $P_{3}$ is slightly larger in size than prisms $P_{1}$ and $P_{2}$. Even though it is not necessary that $P_{1}$ and $P_{2}$ be identical, we have made them identical, as shown in Fig. 4. To avoid errors in the formation of the angle 55-deg, 44min, 8-sec due to nonuniform bevels at the prism edges $E$ (in Fig. 3), we have made them by sticking well-polished plane parallel glass plates in-between waste plane parallel glass plates on either side using a mixture of beeswax and rosin, as shown in Fig. 4. The right angles, pyramidal errors of $P_{1}, P_{2}$, and $P_{3}$, are tested and corrected as shown in Fig. 2 , and 45-deg angles by the method shown in Fig. 5. The error in the measurement of the 45-deg angle is given by

$\beta=\frac{A B}{4 O C}$.

The detailed discussion of these experiments is given by the author in Refs. 8, 11, and 12.

After the separation and cleaning of prisms $P_{1}$ and $P_{2}$, they are joined to the faces opposite the 45-deg angles of prism $P_{3}$ (which is placed on an optical plane surface not shown in Fig. 3) by wringing with drops of alcohol. An optical block of suitable thickness and a straight end is brought in contact with the bottom ends of $P_{1}, P_{3}$, and $P_{2}$ (at the corners $C_{1}$ ) to set them in a single straight line. After the adjustment, a small amount of beeswax is placed

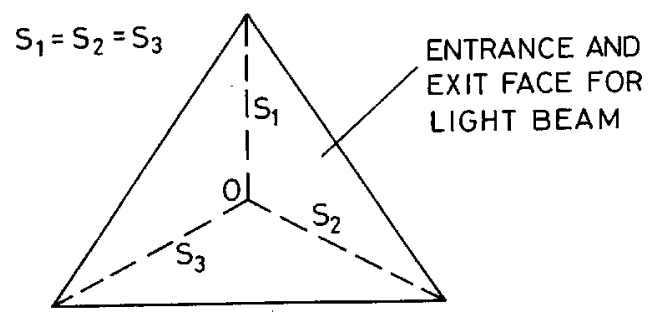

Fig. 1 Schematic diagram of a tetragonal prism (corner cube). 


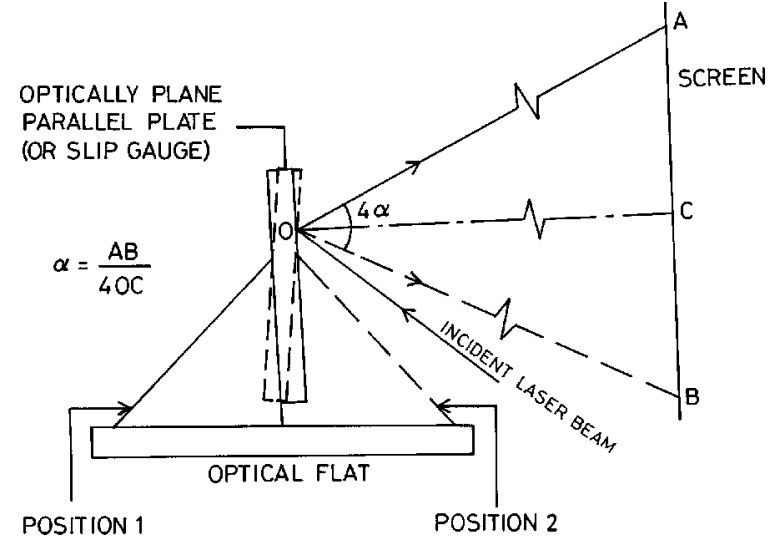

Fig. 2 Schematic diagram showing the principle for testing the 90deg angle of a prism. The angle $4 \alpha$ is greatly exaggerated in this diagram.

on $P_{1}, P_{2}$, and $P_{3}$ in few places so that $P_{3}$ can hold $P_{1}$ and $P_{2}$. Finally, a plane parallel plate (or slip gauge) $R$ is placed on $P_{1}$ and $P_{2}$, as shown in Fig. 3. A little amount of beeswax is placed in some places of $P_{1}, P_{2}$, and $R$ to hold $R$ by $P_{1}$ and $P_{2}$. Now the reference surface $\mathrm{R}$ of this assembly (Fig. 3) makes an angle of 54-deg, 44-min, 8-sec with the base plate on which this arrangement stands.

The test for the 54-deg, 44min, 8-sec angle is performed as shown in Fig. 6. Initially a collimated laser beam is made to incident on the reference surface $R$ of Fig. 3. The position of the reflected laser spot on the screen is noted as $A$, and then the whole arrangement shown in Fig. 3 is replaced by the tetragonal prism to be tested. The reflected laser beam from the surface of the tetragonal prism strikes at $B$ on the screen. Point $C$ is the midpoint of $\mathrm{AB}$. If the error in the tetragonal prism angle 54-deg, $44-\mathrm{min}, 8$-sec is $\gamma$, it is given by

$\gamma=\frac{A B}{2 O C}$.

The details about the measurement of $\alpha, \beta$, and $\gamma$ can be found in Refs. 7-12. In the case of fully polished components, a layer of grease must be applied to the rear surfaces

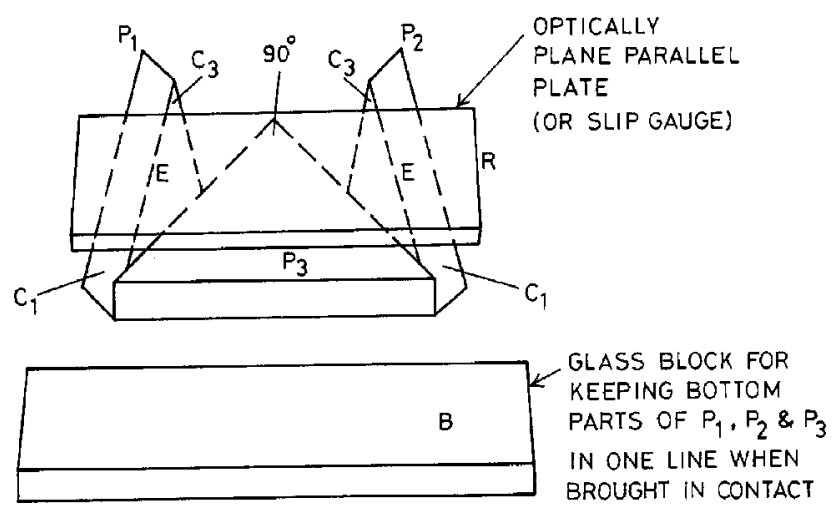

Fig. 3 Schematic diagram illustrating the method of setting the reference surface $R$ for testing the 54-deg, 44-min, 8-sec angle of a tetragonal prism.

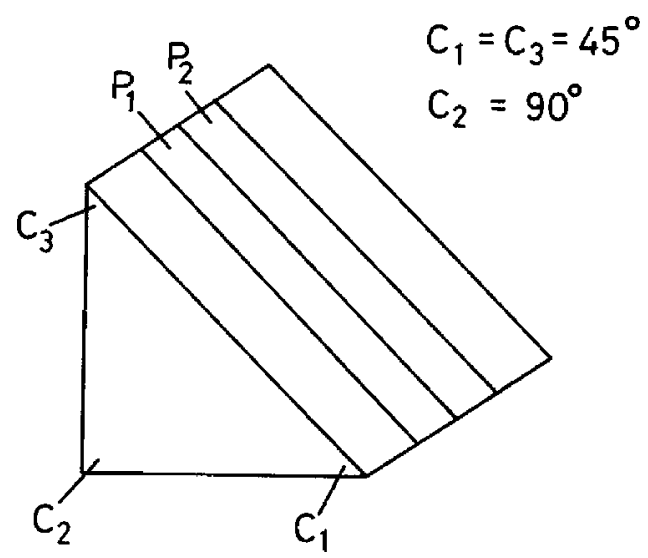

Fig. 4 Schematic diagram of the method for fabrication of right angle prisms $P_{1}$ and $P_{2}$.

of the optical components to avoid the unwanted reflected light coming from some other surfaces. In the case of unpolished components, these experiments must be performed by placing a plane parallel reflecting surface on the test component to reflect the laser beam. The accuracy in the measurements depends on the distance $O C$ (Figs. 2, 5, and 6) of the screen from the point of incidence of the laser beam on the optical component. For this case $O C=5 \mathrm{~m}$. The distances $O C$ are measured to an accuracy of $1 \mathrm{~mm}$. A method of increasing the screen distance is given in Ref. 9. The distances $A B$ are measured using a micrometer eyepiece to an accuracy of $0.01 \mathrm{~mm}$. The error in the measurement of $\alpha, \beta$, and $\gamma$ is less than $1 \mathrm{sec}$ of an arc.

The error in the measurement of any one right angle of the tetragonal prism is less than $1 \mathrm{sec}$ of an arc, but for the angle 54-deg, 44-min, 8-sec, the expected accuracy is less than $5 \mathrm{sec}$ of an arc, provided the components $P_{1}, P_{2}, P_{3}$, and $R$ (Fig. 3) are all well corrected. The accuracy can further be improved by repeating the experiment by placing

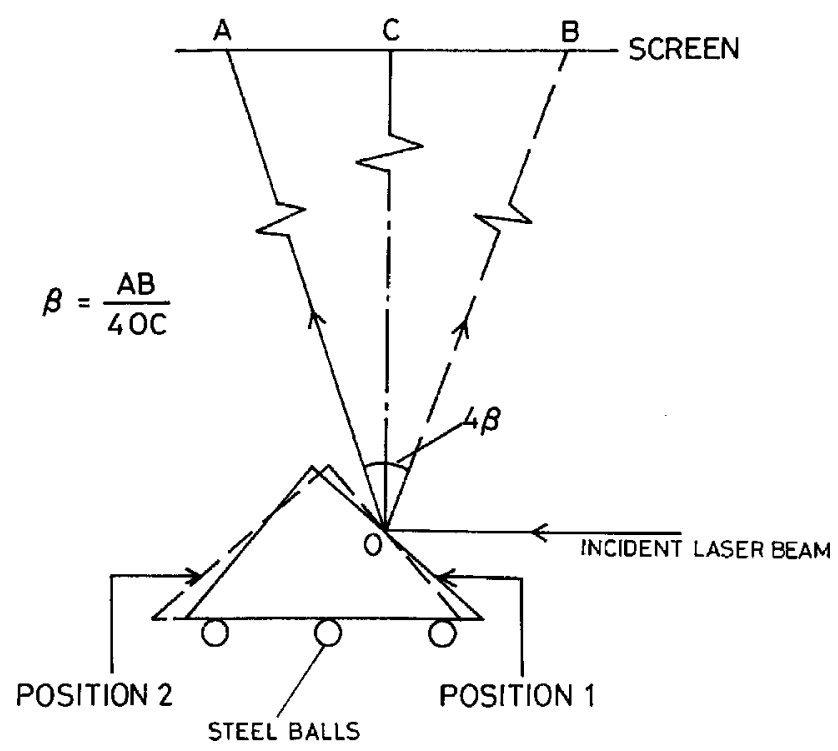

Fig. 5 Schematic diagram showing the principle of testing the 45deg angle of a prism. The angle $4 \beta$ is greatly exaggerated in this diagram. 
Rao: Method for measurement ...

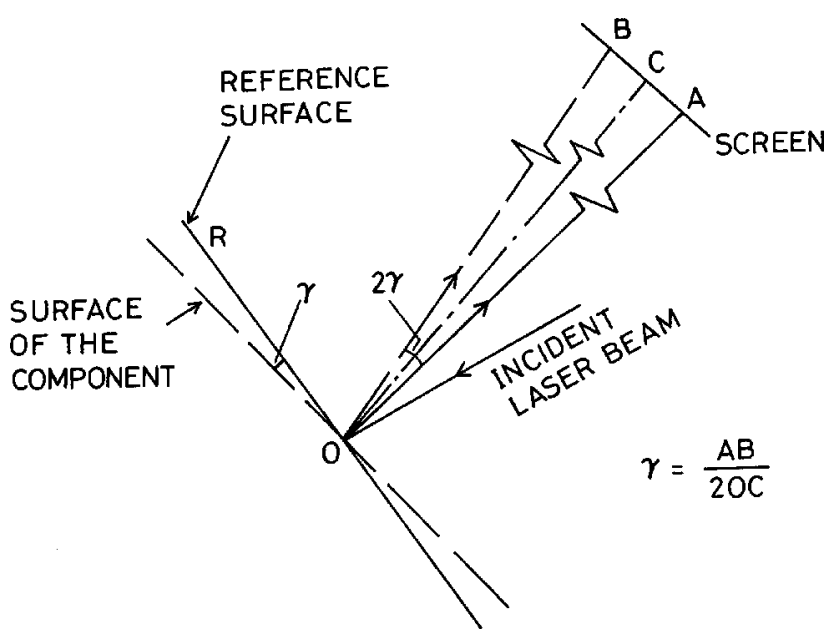

Fig. 6 Schematic diagram showing the principle of testing the 54deg, 44-min, 8-sec angle of a tetragonal prism. The angle $2 \gamma$ is greatly exaggerated in this diagram.

two more sides of the tetragonal prism on the horizontal base plate (used for resting the assembly of Fig. 3 and the tetragonal prism surface) and further polishing the fourth surface of the prism until the errors $\gamma$ become smaller and equal with respect to all three sides. The 54-deg, 43-min, 12 -sec angles of Fresnel rhombs made up of borosilicate crown glass $\left(n_{d}=1.511\right)$ can also be tested against $R$ shown in Fig. 3.

The experiments suggested (in Figs. 2, 5, and 6) can also be performed using an autocollimator when suitable angle gauges are not available.

\section{Conclusions}

The proposed method does not require precision spectrometers, or autocollimators and standard angle gauges. It is a simple, accurate, and relatively inexpensive method.

\section{Acknowledgment}

The author wishes to thank Prof. S. Mohan, Prof. M. V. Krishnamurthy, and Dr. K. Narasimha Rao for useful discussions while conducting these experiments.

\section{References}

1. C. C. Deve, Optical Workshop Principles, 2nd ed., pp. 263-280, Hilger and Watts, London (1954).

2. F. Twyman, Prism and Lens Making, 2nd ed., pp. 300-303, Hilger and Watts, London (1957)

3. A. S. De Vany, Master Optical Techniques, pp. 156-170, 485, John Wiley and Sons, New York (1981).

4. D. F. Horne, Optical Production Technology, pp. 343-351, Adam Hilger, London, (1972).

5. B. K. Johnson, Optics and Optical Instruments, pp. 159-200, Dover Publications, New York (1960)

6. D. Malacara, Optical Shop Testing, pp. 1-79, John Wiley and Sons, New York (1978).

7. M. V. R. K. Murty and R. P. Shukla, "Method for measurement of parallelism of optically parallel plates," Opt. Eng. 18(3), 352-353 (1979).

8. S. Madhusudana Rao, "Method for measurement of the angles of 90-deg, 45-deg, 45-deg and 60-deg, 30-deg, 90-deg prisms," Opt. Eng. 36(1), 198-200 (1997).

9. S. Madhusudana Rao, "Method for measurement of the angles of an equilateral (60-deg) prism," Opt. Eng. 36(5), 1508-1509 (1997).

10. S. Madhusudana Rao, "Method for measurement of the angles of polygons," Opt. Eng. 36(7), 2062-2067 (1997).

11. S. Madhusudana Rao and K. Narasimha Rao, "Method for measurement of the angles of a pentaprism," Opt. Eng. 37(4), 1368-1371 (1998).

12. S. Madhusudana Rao and K. Narasimha Rao, "Method for measurement of the angles of a 45-deg deflecting (half penta) prism," Opt. Eng. 39(6), 1573-1575 (2000)

13. S. M. Rao, "Methods for making prisms with sub-multiple or half angles: applications to the measurement of the angles of Pechan and Pellin-Broca prisms," Opt. Eng. (in press).

S. Madhusudana Rao received his MSc degree in nuclear physics from Andhra University at Waltair, India, in 1972, and began working in the field of testing and calibration of optical instruments with the Andhra Scientific Company (presently BEL) in Masulipatam. In 1977 he joined the Indian Institute of Science, Bangalore, where he received MSc (Eng) and PhD degrees in 1994 and 2000. He is now a Principal Research Scientist in the Department of Instrumentation. $\mathrm{He}$ has several research publications to his credit. His research interests include optical instrumentation, stress analysis, and image processing. He is a member of the Instrument Society of India. 